\title{
Revised molecular phylogeny of Acrididae family
}

\author{
I. Sukhikh ${ }^{1 *}$, K. Ustyantsev ${ }^{1}$, V. Vavilova ${ }^{1}$, A. Blinov ${ }^{1,2}$ \\ ${ }^{1}$ Institute of Cytology and Genetics SB RAS, Novosibirsk, Russia \\ ${ }^{2}$ Institute of Immunology and Physiology UB RAS, Yekaterinburg, Russia \\ *e-mail: igor3419@gmail.com
}

Key words: Acrididae, phylogeny, grasshoppers, mitochondrial DNA, ribosomal DNA

Motivation and Aim: Acrididae is the biggest family of Acridoidea superfamily, consisting of more than 6000 species out of 12000 species in the Caelifera suborder. Different authors distinguish different number of subfamilies, reaching the limit of 33. It is worth noting that the few subfamilies consists of small number of genus and species. For a long time, taxonomy, the renovation of phylogenetic relationships and understanding of the evolution of Acrididae family were based mainly on comparison analysis of key morphological structures of recent and fossil species. Currently, one of the most effective methods for establishing phylogenetic relationships is the analysis of mitochondrial and ribosomal DNA. For grasshoppers, there are 4 most widely used phylogenetic markers mitochondrial genes COI, COII, Cytb and NADH5, as well as sequences of nuclear (18S, 28S, including ITS sequences) and mitochondrial (12S, 16S) clusters of ribosomal genes. In the present study, we used markers with the most overlapping species and aimed to include nuclear and mitochondrial DNA.

Methods and Algorithms: New nucleotide sequences of COI, COII and ITS2 markers of Acrididae species were obtained using Sanger sequencing and uploaded in GenBank NCBI database. Phylogenetic trees were built using Maximum likelihood and Bayesian methods.

Results: In present study, we performed phylogenetic analysis of more than 240 species of 14 subfamilies of Acrididae family, based on complete mitochondrial DNA sequences, concatenated DNA sequences of CytB, COII and COI genes, concatenated DNA sequences of COII and COI genes and DNA sequences of ITS2 region. Phylogenetic tree constructed using complete mitochondrial sequences served as a basis for the analysis. Sequences of studied Acrididae species divide into two major groups, which in turn divides into several clusters, as well as four separate branches, each consisting of species of single subfamily (Catantopinae, Oxyinae, Spathosterninae and Proctolabinae). First group consists of representatives of three subfamilies: Acridinae, Gomphocerinae and Oedipodinae; second group includes 8 subfamilies: Catantopinae, Calliptaminae, Conophyminae, Cyrthacantacridinae, Eyprepocnemidinae, Hemiacridinae, Melanoplinae and Pezotettiginae. Five out of 14 subfamilies (Acridinae, Oedipodinae, Gomphocerinae, Oxyinae and Catantopinae) found to be polyphyletic in the present study. Geographical distribution of Acrididae species in polyphyletic branches in most cases correlate with their positions on the phylogenetic tree.

Conclusion: The results obtained show that the current systematic of Acrididae family requires revision of at least five subfamilies, which were found to be polyphyletic. All experimentally obtained sequences of Acrididae species are available in the NCBI GenBank database under accession numbers KX272717-KX272739 for COI gene, KX272670-KX272716 for COII gene and KX289534-KX289579 for ITS2 region. Acknowledgements: The project was supported by the Russian Foundation for Basic Research (Grant No. 17-04-01615). 